\title{
FDG-PET positive pilomatrixoma - reconsidering multicentricity in Langerhans cell histiocytosis
}

Petr Szturz ${ }^{1}$, Zdeněk Řehák², Renata Koukalová2, Zdeněk Adam¹, Jiří Mayer ${ }^{1}$

${ }^{1}$ Department of Internal Medicine, Hematology and Oncology, University Hospital Brno and Masaryk University, School of Medicine, Czech Republic

2Department of Nuclear Medicine, PET Center, Masaryk Memorial Cancer Institute, Brno, Czech Republic

[Received 22 XI 2013; Accepted 7 VII 2014]

\begin{abstract}
We report a case of a woman who underwent a curative resection of an upper jaw tumor histologically verified as eosinophilic granuloma. To exclude possible multiorgan involvement, PET/CT imaging was performed and revealed a metabolically active, partially calcified lesion located on the chest wall surface and clinically corresponding to a gradually developing, round, subcutaneous infiltrate with erythematous overlying skin. After complete extirpation, the pathological finding was consistent with pilomatrixoma surprisingly, thus dismissing the suspected diagnosis of multi-system Langerhans cell histiocytosis.
\end{abstract}

KEY words: Langerhans cell histiocytosis, pilomatrixoma, positron emission tomography

Nuclear Med Rev 2014; 17, 2: 94-96

\section{Introduction}

Defined either as single-system or multi-system form, Langerhans cell histiocytosis encompasses diverse clinical manifestations with various treatment approaches and a wide prognostic spectrum; hence the name sometimes used to characterize the disease - chameleon-like. Virtually any part of the body may be affected, though most commonly involved are the skeletal system, skin and lungs [1]. We report a case of a woman with Langerhans cell histiocytosis demonstrating a diagnostic pitfall in fluorine-18-fluorodeoxyglucose (FDG) positron emission tomography/computed tomography (PET/CT) scan imaging when searching for potential multicentric disease involvement.

\section{Case report}

A 67-year-old female, born in 1946, was referred to our department for further evaluation after resection of an upper jaw tumor at a dentistry clinic. Histopathological examination showed eosinophilic granuloma, a localized bone form of Langerhans cell histiocytosis. In order to exclude possible multifocal bone affection or involvement of other organs, the patient underwent PET/CT scan

Correspondence to: Petr Szturz, MD, PhD

Department of Internal Medicine, Hematology and Oncology

University Hospital Brno

Jihlavská 20, 62500 Brno, Czech Republic

Phone: +420532232934

Fax: +420532233603

E-mail: petr.szturz@fnbrno.cz imaging revealing no residual hypermetabolism in the postresection area. However, a metabolically active (Maximum Standardized Uptake Value $=3.86$, Figure 1 and 2, see red arrows), partially calcified lesion (14 mm in diameter, Figure 3, see blue arrow for the calcification) was identified located on the chest wall surface ventrolaterally at the level of the 7th rib-cartilage junction. Clinically, the infiltrate corresponded to a round subcutaneous lump with erythematous overlying skin, which had been slowly developing over a period of the past 3 years (Figure 4, see yellow arrow). After complete extirpation, the pathological finding was consistent with benign pilomatrixoma surprisingly, thus dismissing the suspected diagnosis of multi-system form of Langerhans cell histiocytosis. At 6 months after the surgical procedures, follow-up examinations (clinical, dental panoramic radiograph) showed a sustained complete remission.

The PET/CT scanning was performed in euglycemia (blood glucose level $=5.2 \mathrm{mmol} / \mathrm{l}$ ) after fasting for 6 hours and an adequate oral pre-hydration with $500 \mathrm{~mL}$ of water. The scans were obtained after 60 min accumulation phase following intravenous application of FDG (UJV Rez, Czech Republic) in the dose of 314 MBq. Data acquisition on a hybrid PET/CT scanner (Biograph 64 True Point $H R$, Siemens, Erlangen, Germany) was carried out in two phases: (i) at the extent from the cranial base to the upper thighs with a high-dose contrast enhanced CT using $70 \mathrm{ml}$ of intravenous iodine radiocontrast (Iomeron 400, Bracco, Milano, Italy), (ii) examination of the remaining lower extremities with a low dose non-contrast CT. Emission and transmission scans were reconstructed by using an iterative reconstruction algorithm. We evaluated PET scans, CT scans as well as fused PET/CT images. For semiquantitative analysis, SUVmax values were measured. The PET reconstruction 
parameters were as follows: iterative reconstruction algorithm, OSEM, 4 iterations, 8 subsets, image size 168, zoom 1, Filter Gaussian, FWHM 6mm. The acquisition parameters for CT were as follows: slice $3 \mathrm{~mm}$, Kernel B30f, Filter "PET medium smooth", FoV $500 \mathrm{~mm}$, reconstruction increment $3 \mathrm{~mm}$.

\section{Discussion}

Being an uncommon idiopathic disorder, Langerhans cell histiocytosis is characterized by proliferation and accumulation of clonal

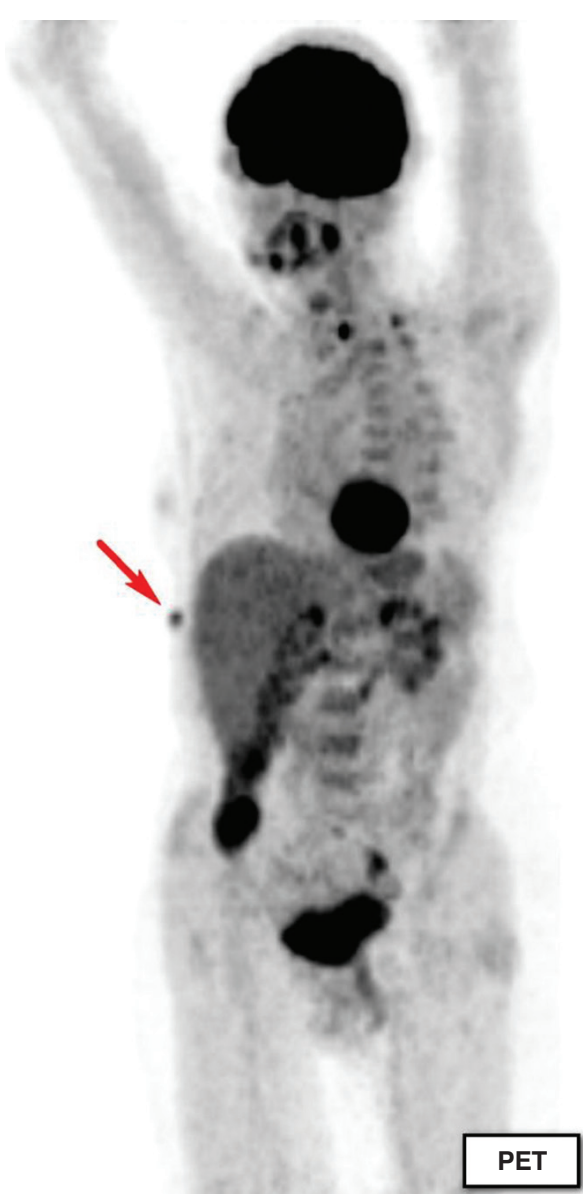

Figure 1. PET imaging, Maximum Intensity Projection. See red arrow for the metabolically active infiltration



Figure 3. CT imaging, coronal scan. See blue arrow for the calcification

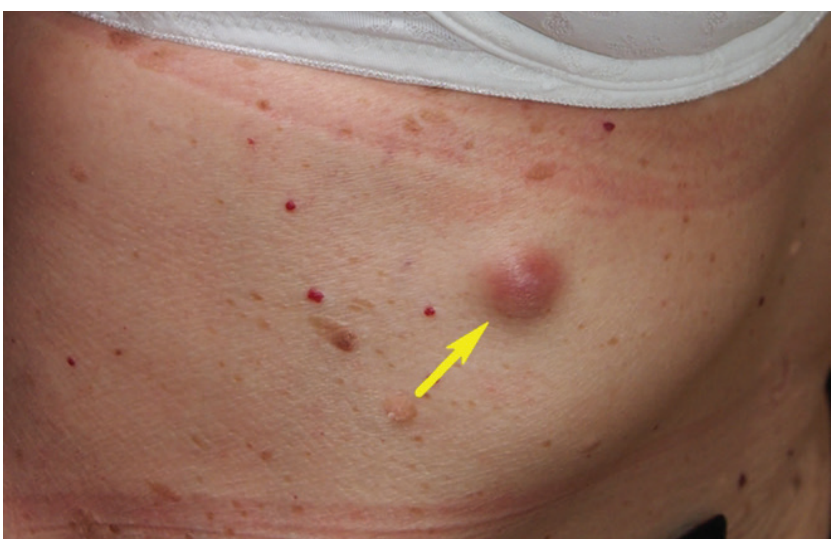

Figure 4. See yellow arrow for the clinical correlate

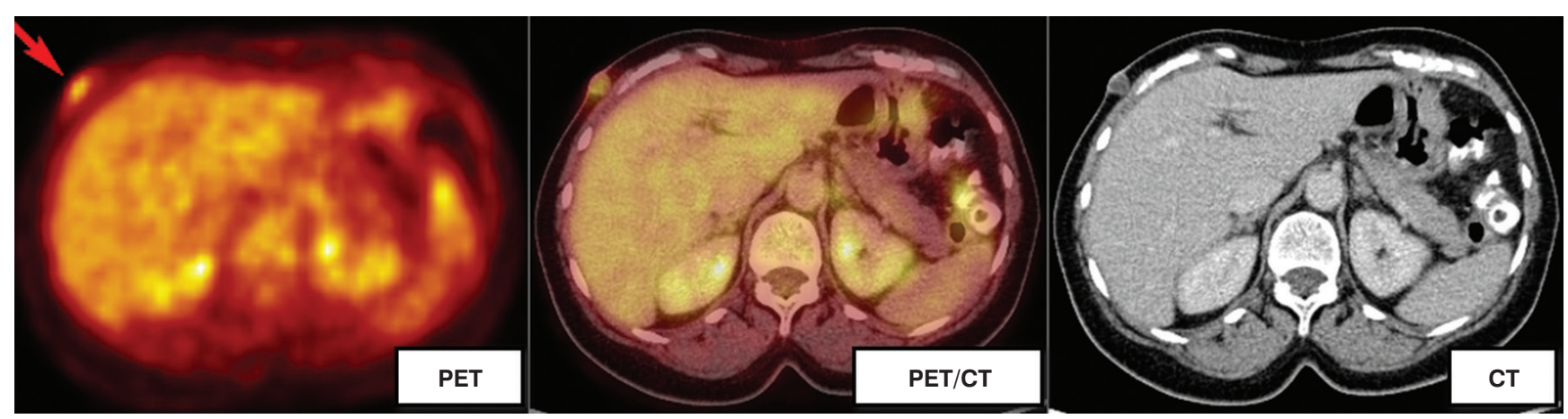

Figure 2. PET/CT imaging, axial scans. See red arrow for the metabolically active infiltration 
Langerhans cells in various tissues and organs resulting in abundant clinical manifestations ranging from a single osteolytic lesion (eosinophilic granuloma) to widespread disease with multiorgan involvement. A recent observation of a high frequency of activating BRAFV600E mutations in lesional cells supports its classification as a neoplastic histiocytic condition [1]. Pilomatrixoma or calcifying epithelioma of Malherbe represents a rare benign skin appendageal tumor usually affecting the head and neck regions in children with a female predominance. Complete surgical excision as a potentially curative modality may fail in cases of a far less common malignant variant [2]. A differential diagnosis comprises varieties of benign (e.g. sebaceous cyst, giant cell tumor, foreign body reaction) as well as malignant (e.g. basal cell carcinoma) conditions. These are not always possible to be reliably distinguished by fine needle aspiration biopsy or imaging methods [3]. Therefore, a histopathological evaluation is imperative.

A June 2013 MEDLINE literature search using the keywords piIomatrixoma and positron emission tomography retrieved two articles reporting on three adult patients with a solitary, metabolically active mass located in the head and neck area and initially mistaken for a carcinoma according to fine needle biopsy $[4,5]$. The herein presented case of unicentric Langerhans cell histiocytosis coinciding with FDG-PET positive pilomatrixoma demonstrates a possible oncologic pitfall for the clinicians underlined by the rarity of both these entities.

\section{Acknowledgement}

This work was supported in part by project MUNI/A/0723/2012, by $\mathrm{MH} \mathrm{CZ}$ - DRO (MMCl, 00209805), MH CZ — DRO (FNBr, 65269705) and OP VaVpl - RECAMO.

\section{References}

1. Girschikofsky M, Arico M, Castillo D, Chu A, Doberauer C, Fichter J et al. Management of adult patients with Langerhans cell histiocytosis: recommendations from an expert panel on behalf of Euro-Histio-Net. Orphanet J Rare Dis 2013; 8: 72

2. Pirouzmanesh A, Reinisch JF, Gonzalez-Gomez I, Smith EM, Meara JG. Pilomatrixoma: a review of 346 cases. Plast Reconstr Surg 2003; 112: 1784-1789.

3. Kumar N, Verma K. Fine needle aspiration (FNA) cytology of pilomatrixoma. Cytopathology 1996; 7: 125-131.

4. Jung YS, Kang JG, Park WS, Ryu J. Pilomatricoma: diagnostic pitfalls in PET/CT and fine-needle aspiration biopsy. Otolaryngol Head Neck Surg 2007; 137: 845-846.

5. Bhatt MK, Sommerville R, Ravi Kumar AS. FDG PET/CT appearance of benign pilomatricoma. Clin Nucl Med 2012; 37: 684-686. 